\title{
Selected and Critical Abstracts on
}

\section{- Tropical diseases - Communicable diseases - Environmental health - Community health}

Each month the Bureau of Hygiene and Tropical Diseases publishes two complementary journals of selected and critical abstracts of relevant papers published in over 1100 journals, as well as book reviews, authoritative review articles and citations of governmental and other reports.

Both journals are available on magnetic tape.

\section{- Tropical Diseases Bulletin}

emphasizes those aspects of particular relevance to tropical and subtropical countries and includes sections on medical parasitology, medical entomology, insecticides, venoms, and community health, in addition to the main sections on tropical and communicable diseases.

\section{- Abstracts on Hygiene and Communicable Diseases covers aspects of health and disease of worldwide importance and includes sections on health services, food hygiene and poisoning, environmental pollution, as well as the major sections on bacterial, viral and other communicable diseases.}

For subscription prices and further information please write to The Director, Bureau of Hygiene and Tropical Diseases, Keppel Street, London WC1E 7HT, U.K. A free copy of either publication is available on request. 